\title{
Tierseuchen und One Health
}

\author{
Thomas C. Mettenleiter ${ }^{(\varpi)}$ \\ Friedrich-Loeffler-Institut, Bundesforschungsinstitut für Tiergesundheit (FLI), \\ Greifswald-Insel Riems, Deutschland \\ ThomasC.Mettenleiter@fli.de
}

Schlüsselwörter: Tierkrankheiten · COVID-19 · One Health

\section{Das One Health Konzept}

„Zwischen Tier- und Menschenarzneikunde ist oder sollte wissenschaftlich keine Scheidegrenze sein. Das Objekt ist verschieden, aber die Erfahrungen, die aus dem Objekt zu schöpfen sind, sind Lehrsätze, welche die Grundlage der Doktrinen bilden." Dieses Zitat von Rudolf Virchow von 1873 steht am Beginn der Entwicklung, die zum Konzept „One Health“ geführt hat. 1964 von Schwabe in einem Lehrbuch aufgegriffen, prägte er den Begriff „One Medicine“. One Health erweitert One Medicine um den Aspekt der Umwelt: Der Mensch ist Teil des Tierreichs in einer gemeinsamen Umwelt [1]. Dies wurde von der Wildlife Conservation Society in 2004 in den „Manhattan Principles“ erstmals niedergelegt [2] und kürzlich in den ,Berlin Principles' nochmals erweitert [3]. One Health beschreibt demnach die Verbindung zwischen der Gesundheit von Mensch, Tier und Umwelt. Dies kann in drei Kreisen dargestellt werden, die bilaterale Überlappungen aufweisen, aber in einer zentralen Schnittmenge den eigentlichen Bereich One Health umfassen (Abb. 1). Diese Schnittmenge wird durch das Wachstum der menschlichen Population, die zunehmende Nachfrage nach Nahrung, darunter tierisches Protein, und die damit zusammenhängende Beeinflussung der Umwelt ständig umfangreicher. One Health bezeichnet also die Schnittstelle aller drei Sektoren, wobei die anderen Schnittstellen nicht außer Acht gelassen dürfen: die Schnittstelle Mensch-Tier als One Medicine, die Schnittstelle Tier-Umwelt und die Schnittstelle Mensch-Umwelt. 


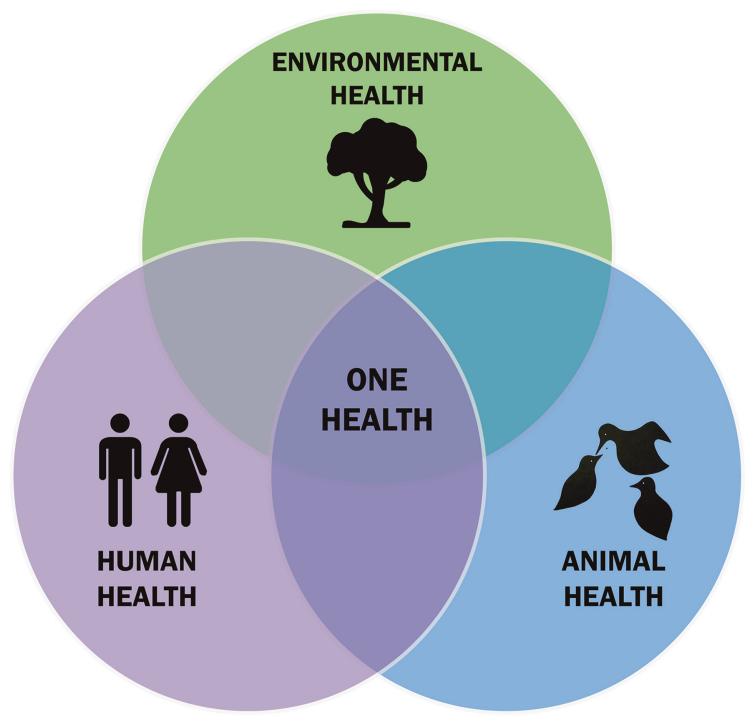

Abb. 1. Das One Health Konzept. (Thddbfk/Wikimedia Commons)

\section{Zoonosen}

Im Zentrum von One Health im Kontext von Infektionen stehen antimikrobielle Resistenzen und Zoonosen. Als Zoonosen werden Infektionen bezeichnet, die auf natürliche Weise zwischen Menschen und anderen Wirbeltieren übertragen werden (Abb. 2). Das ist eine Definition der Weltgesundheitsorganisation WHO, die aus dem Jahr 1954 stammt und somit recht alt, aber eindeutig ist. Der Begriff ,Zoonose" beinhaltet dabei keine Richtung. Er beschreibt also die bidirektionale Infektion von Tier zu Mensch und von Mensch zu Tier. Direktionalität enthalten die Termini ,Anthropozoonose“ (von Mensch zu Tier) beziehungsweise ,Zooanthroponose“ (von Tier zu Mensch).

Gerade in den letzten Wochen wurde immer wieder gefragt, wie es dazu kommt, dass Erreger plötzlich vom Tier auf den Menschen überspringen. Die Gegenfrage ist: Wieso denn nicht? Der Begriff Zoonose basiert darauf - und es ist erstaunlich, wie wenig das im Bewusstsein vieler Menschen verankert ist -, dass der Mensch biologisch Teil des Tierreichs ist und damit keine besondere natürliche Barriere zwischen Mensch und Tier existiert. So gesehen verwundert es nicht, dass viele der humanen Infektionen auf Erregern basieren, die aus dem Tierreich stammen. 


\section{Zoonotic diseases and human spread}

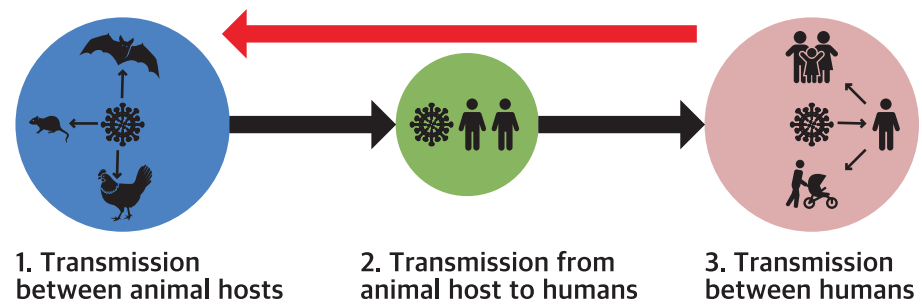

Abb. 2. Spill-Over und Ausbreitung von Zoonosen. (Eigene Darstellung nach World Health Organization)

Etwa $60 \%$ der menschlichen Infektionskrankheiten sind tierischen Ursprungs und etwa $75 \%$ der „Emerging Infections“, also der neu auftretenden Infektionskrankheiten, sind Zoonosen. So gehen beispielsweise die Erreger von Rinderpest und Masern auf einen gemeinsamen Vorläufer zurück. In historischen Zeiträumen haben sie sich dann voneinander getrennt [4]: Die Masern sind heute eine rein humane Infektion, die Rinderpest eine reine Tierseuche. Und erst kürzlich konnten Arbeitsgruppen aus den USA und am Friedrich-Loeffler-Institut zwei Verwandte des menschlichen Röteln-Virus in tierischen Reservoiren identifizieren, in Fledermäusen in Afrika und bei Gelbhalsmäusen in Nordost-Deutschland [5].

An den 13 wichtigsten Zoonosen sterben jährlich durchschnittlich rund 2,2 Mio. Menschen. Im Mittel erkranken pro Jahr rund 2,4 Mrd. Menschen - also jeder Dritte der Weltbevölkerung - an einer dieser zoonotischen Infektionen. Zu den 13 wichtigsten Zoonosen gehören zuvorderst gastrointestinale Infektionen, die zu einem großen Teil parasitäre Ursachen haben. Dazu kommen dann weitere sogenannten „Neglected Tropical Diseases“ (vernachlässigte Tropenkrankheiten), wie zum Beispiel die Tollwut. Die Krankheitslast ist dabei in weniger entwickelten Ländern am höchsten.

\section{COVID-19, die neu aufgetretene Zoonose}

COVID-19, der Erreger der gegenwärtigen Pandemie, stammt wohl aus einem tierischen Reservoir aus Südchina. Höchstwahrscheinlich sind Hufeisennasen-Fledermäusen die ursprünglichen Wirte. Wann, wie und wo die Viren auf den Menschen übertragen wurden, ist allerdings bisher nicht bekannt. Außerdem unklar ist, ob - wie bei SARS-CoV - tierische Brückenwirte eine Rolle gespielt haben. Nach dem Übertritt in die menschliche Population hat sich der Erreger zunächst lokal massiv ausgebreitet und in der Folge durch den internationalen Reiseverkehr zur vom Menschen getragenen Pandemie geführt.

Verschiedene Tierarten sind empfänglich für den COVID-19-Erreger [6] und infizierte Menschen tragen ihn wieder zurück in das Tierreich. Er kann von diesen 
infizierten Tieren - glücklicherweise bisher nur in seltenen Fällen - wieder auf den Menschen zurück übertragen werden. Dies zeigt eine Situation wie bei vielen Zoonosen: ein Überspringen aus dem tierischen Reservoir auf den Menschen, Anpassung an den Menschen und nachfolgend die epidemische oder pandemische Ausbreitung mit einer Rückübertragung auf empfängliche Tiere.

Es gilt, diesen Infektionszyklus zu blockieren. So sollte vermieden werden, unsere Haustiere mit COVID-19 zu infizieren. Es gibt weltweit etwa siebzig gut dokumentierte Fälle von Infektionen von Haustieren durch COVID-19-infizierte Menschen, etwa zwei Drittel davon Katzen, ein Drittel Hunde. Auch Zootiere Großkatzen und Primaten - wurden in Einzelfällen infiziert. Durch den Eintrag des Virus in Nerzpopulationen, die zur Pelzgewinnung gehalten werden, haben sich regionale Epidemien entwickelt, insbesondere in den Niederlanden und in Dänemark. Diese wurden mit den Mitteln der Tierseuchenbekämpfung bekämpft. Dokumentiert ist, dass Menschen durch Nerze, die den COVID-19-Erreger in sich trugen, infiziert wurden [7].

Pelztiere, darunter auch die Marderhunde, sind ein potenziell gefährliches Tierreservoir. Über $99 \%$ der Weltpopulation von Marderhunden, die zur Pelzgewinnung gezüchtet werden, lebt in China. In Deutschland haben wir keinerlei Informationen, was dort geschieht und ob dort Tests durchgeführt wurden. Durch umfangreiche Tierversuchsstudien am Friedrich-Loeffler-Institut ist aber bekannt, dass Marderhunde sehr empfänglich für das Virus sind. Sie können es sehr gut vermehren, ausscheiden und auf Artgenossen übertragen [8]. Alles das, was bei den Nerzen auftritt, kann bei Marderhunden auch geschehen.

\section{Schutz vor künftigen Pandemien}

Was können wir tun, um das Überspringen von Viren aus dem tierischen Reservoir zu verhindern? Hier kommt das One Health Konzept ins Spiel, das in der Zwischenzeit nicht nur bei der Politik angekommen ist, sondern auch bei den Medien: „Das Rezept gegen Killerviren. Ein neuer Ansatz, genannt One Health, soll verhindern, dass sich Pandemien wie Corona wiederholen", stand in der Frankfurter Allgemeinen Zeitung Nr. 219 vom 19.09.2020. Wie berichtet, ist der Ansatz eigentlich nicht neu. Aber heute machen sich offensichtlich mehr Menschen Gedanken darüber. Bewirkt die COVID19-Krise nun ein „Erwachsenwerden“ von One Health [9]?

Ein Beispiel, wie ein Überspringen verhindert werden kann, sozusagen das klassische Beispiel von One Health, ist die Tollwut. Sie beinhaltet die Impfung von Hunden - und in Mitteleuropa später Füchsen -, um dort die Tollwut zu bekämpfen, damit diese Tiere keine Gefahr mehr für den Menschen darstellen. Der Mensch wird also geschützt durch den Schutz der Tierpopulation oder durch Maßnahmen in der Tierpopulation. Besonders spannend ist dabei, dass diese Strategie in diesem Fall sogar in einer Wildtierpopulation gelang.

Allerdings ist es unmöglich, gegen jedes Virus, das im Tierreich vorkommt, einen Impfstoff zu entwickeln. Es gibt Schätzungen, wie viele unerkannte Viren in der tierischen Population - nur den Säugetieren - noch schlummern. Diese reichen von 
rund 300.000 bis zu über 1.000.000 [10]. Häufig richtet sich die Aufmerksamkeit auf exotische Tiere, wie sie etwa auf den Tiermarkt in Wuhan gehandelt werden. Solche Tiermärkte ebenso wie andere Situationen, bei denen viele Individuen und Arten aus unterschiedlichen Regionen zusammenkommen, sind Drehscheiben für den Austausch von Erregern, im Falle von Tiermärkten auch direkt im Kontakt zum Menschen. Und exotische Tiere können exotische Erreger tragen. Beispielsweise wurde am FriedrichLoeffler-Institut vor fünf Jahren bei Bunthörnchen ein neuartiges Bornavirus entdeckt, das beim Menschen tödliche Enzephalitiden hervorrufen kann [11]. Bunthörnchen stammen ursprünglich aus Mittelamerika.

Aber auch hierzulande werden völlig neue, bisher unbekannte Seuchenerreger gefunden, wie etwa das Schmallenberg-Virus, das 2011 zum ersten Mal identifiziert wurde und sich in kurzer Zeit epidemisch über Europa ausgebreitet hat [12]. Glücklicherweise ist es ein reines Tierpathogen. Außer auf Wildpopulationen müssen wir auf die Tiere ein besonderes Augenmerk richten, die uns am nächsten sind, also Haus- und Nutztiere. Dies wird im Report der Intergovernmental Platform on Biodiversity and Ecosystem Services deutlich formuliert: „Es überrascht nicht, dass die überwiegende Mehrheit der Tiere, die in historische Zoonoseereignisse oder aktuelle Zoonosen verwickelt sind, domestizierte Tiere sind, was logisch ist, da die Kontaktraten hoch sind. Das Auftreten einer neuen Wildtier-Zoonose ist extrem selten“, (das, was wir jetzt erleben), ,kann aber sehr bedeutsam sein “ (was wir ebenfalls gerade erleben) [13].

In diesem Kontext sind auch die Grippepandemien in den letzten 100 Jahren anzusprechen, die Spanische, Asiatische und die Hongkong-Grippe sowie die Schweinegrippe H1N1. Die Spanische Grippe stammt höchstwahrscheinlich aus USamerikanischen Schweinebeständen, die Asiatische und die Hongkong-Grippe aus Geflügelhaltungen im Fernen Osten und die Schweinegrippe aus Schweinebeständen wohl aus Mexiko. Die Vogelgrippe H5N1 hat glücklicherweise nicht zu einer Pandemie geführt. Aber hier besteht schon von der Bezeichnung her die Assoziation mit Geflügel.

Zusammengefasst darf man sich also nicht immer nur auf die Wildtiere und den Wildtierhandel in exotischen Ländern konzentrieren, sondern muss auch im Auge behalten, was in Europa und Deutschland passiert. So wird in Europa gerade in Schweineställen auf Virenjagd gegangen. Dies ist wegen des Eintrags der Schweinegrippe durch infizierte Menschen in Schweinebestände nötig. Denn der sozusagen rückübertragene Erreger tauscht mit den bereits vorhandenen Schweineinfluenzaviren Gene aus, sodass sich ständig neue Virusvarianten bilden. Darunter sind solche, die einige der Barrieren bereits übersprungen haben, die der Mensch gegenüber Influenzaviren aufgebaut hat [14-16]. Diese werden als Erreger mit präpandemischem Potenzial bezeichnet. Also ist es nicht nur das Schuppentier, oder was auch immer sich an exotischen Tieren in der Welt befindet, von dem Infektionsgefahren für den Menschen ausgehen, sondern die Übertragung auf den Menschen kann auch hierzulande erfolgen.

Das Ganze ist eingebettet in eine Veränderung der Umwelt, unter anderem durch den Klimawandel. Er hat sehr direkte Auswirkungen auf Flora und Fauna. Beispielsweise wandern neue Stechmückenarten, wie die japanische Buschmücke oder die asiatische Tigermücke, nach Deutschland ein - oder auch Zecken, wie die von der 
Presse „tropische Riesenzecke“ genannte Hyalomma. Hier entwickelt sich neues Vektorpotenzial.

Es hat immer ein Überspringen von Erregern aus dem tierischen Reservoir auf den Menschen und zurück gegeben. Es wird wohl auch künftig nicht zu verhindern sein. Man kann versuchen, die Häufigkeit solcher Übergänge durch die Verringerung von riskanten Kontakten zu reduzieren, aber ausschließen kann man die Übergänge nie. Das heißt, man muss sich darauf konzentrieren, sich bildende Infektionsketten möglichst früh zu identifizieren. Hier spielt die Überwachung von Krankheitsbildern zur Früherkennung eine entscheidende Rolle. Diese syndromische Surveillance ist in den letzten Jahren besser geworden. Im Vergleich zu SARS-CoV, wo es Monate dauerte, bis die Krankheit identifiziert und darauf reagiert wurde, waren es bei COVID-19 nur wenige Wochen. Sehr gut wäre es, wenn man diese Zeit künftig auf wenige Tage verkürzen könnte.

\section{One-Health-Forschungsaktivitäten}

Es gibt in Deutschland und weltweit eine Reihe von Forschungsaktivitäten rund um Zoonosen und One Health. So existiert in Deutschland seit über zehn Jahren ein Netzwerk der Zoonosen-Forscher, die Zoonosen-Plattform (www.zoonosen.net). In Greifswald wird ein Helmholtz-Institut errichtet, das sich mit One Health beschäftigen wird. Es gibt internationale Kooperationen der Ernährungs- und Landwirtschaftsorganisation der Vereinten Nationen FAO, der Weltorganisation für Tiergesundheit OIE und der WHO, in denen es darum geht, One-Health-Ansätze zu realisieren. In einigen dieser Programme ist auch das Umweltprogramm der Vereinten Nationen UNEP dabei. Denn One Health beinhaltet eben nicht nur Tier und Mensch, sondern auch die Umwelt. Die zentrale Komponente - die Interaktion zwischen Pathogen, Wirt und Vektor - ist eingebettet in eine komplexe Situation, in der das Klima, das Habitat, die Umwelt, die Globalisierung und Populationen eine Rolle spielen. Eingebettet ist One Health in weitergehende Konzepte von Eco Health bis hin zum Planetary Health.

Die COVID-19-Pandemie hat aber gezeigt, dass das One-Health-Konzept noch nicht überall angekommen ist. So wurde beispielsweise immer wieder artikuliert, dass es keine Impfstoffe gegen Corona gäbe. Bezogen auf SARS-CoV und COVID19 stimmte das anfänglich auch. Doch in der Tiermedizin existieren Impfstoffe gegen Coronaviren schon seit Jahrzehnten und es gibt umfangreiche Erfahrungen mit ihrem Einsatz.

In diesem Kontext wurde auch diskutiert, inwieweit die Expertise der Tiermedizin in die Bekämpfung der COVID-19-Pandemie einbezogen werden kann. Zu ihrem Beginn gab es Diskussionen um die Testkapazitäten. Wahrscheinlich waren damals keine Prüflaboratorien besser für die Seuchendiagnostik ausgerüstet als die tiermedizinischen Labore. Trotzdem gab es Nachfragen, ob diese Labore überhaupt zur Testung im Humanbereich in der Lage wären, ob sie akkreditiert wären und ob sie mit 
Erregern höherer Risikogruppen umgehen könnten. Erst mit dem „dritten Gesetz zum Schutz der Bevölkerung bei einer epidemischen Lage von nationaler Tragweite“ im November 2020 werden die Veterinäre offiziell Teil des Testsystems in Deutschland. Das zeigt, dass in der Umsetzung von One Health in Deutschland noch deutlicher Optimierungsbedarf besteht. Mit einem Augenzwinkern sei gesagt:

- Es ist interessant, dass auch Humanmediziner den Begriff der Herdenimmunität verwenden. Das zeigt, dass das One-Medicine/One-Health-Konzept offenbar doch seine Spuren hinterlässt, denn Menschen leben nicht in Herden.

- Wir sind im Hinblick auf die Realisierung von One Health dann einen Riesenschritt weiter, wenn Humanmediziner erkennen, dass sie im Kern eigentlich „Fachtierärzte für Menschen“" sind.

\section{Literaturs}

1. Zinsstag, J., Schelling, E., Waltner-Toews, D., Tanner, M.: From „one medicine“ to „one health" and systemic approaches to health and well-being. Prev. Vet. Med. 101, 148-156 (2011)

2. http://www.oneworldonehealth.org/sept2004/owoh_sept04html. Zugegriffen: 19. Jan 2021

3. Grützmacher, K. et al.: The Berlin principles on one health - Bridging global health and conservation. Sci. Total Environ. (in Druck)

4. Düx, A., et al.: Measles virus and rinderpest virus divergence dated to the 6th century BCE. Science 368, 1367-1370 (2020)

5. Bennett, A.J., et al.: Relatives of rubella virus in diverse mammals. Nature 586, 424-428 (2020)

6. Schlottau, K., et al.: SARS-CoV-2 in fruit bats, ferrets, pigs, and chickens: an experimental transmission study. Lancet Microbe 1, e218-e225 (2020)

7. Oude Munnink, B.B. et al.: Transmission of SARS-CoV-2 on mink farms between humans and mink and back to humans. Science 371, 172-177 (2020)

8. Freuling, C., et al.: Susceptibility of raccoon dogs for experimental SARS-CoV-2 infection. Emerg. Infect. Dis. 26, 2982-2985 (2020)

9. de Garine-Wichatitsky, M., et al.: Will the COVID-19 crisis trigger a One Health coming of age? Lancet Planet Health 4, e377-e378 (2020)

10. Anthony, S.J., et al.: A strategy to estimate unknown viral diversity in mammals. mBio 4, e00598-e00613 (2013)

11. Hoffmann, B., et al.: A variegated squirrel bornavirus associated with fatal human encephalitis. N. Engl. J. Med. 373, 154-162 (2015)

12. Hoffmann, B., et al.: Novel orthobunyavirus in cattle, Europe, 2011. Emerg. Infect. Dis. 18, 469-472 (2012)

13. UN Environment Program and International Livestock Research Institute: Preventing the next pandemic: Zoonotic diseases and how to break the chain of transmission. Spec. UNEP's Frontiers Rep. Ser. (2020) 
14. Everett, H.E., et al.: Interspecies transmission of reassortant swine influenza A virus containing genes from Swine Influenza $\mathrm{A}(\mathrm{H} 1 \mathrm{~N} 1) \mathrm{pdm} 09$ and $\mathrm{A}(\mathrm{H} 1 \mathrm{~N} 2)$ viruses. Emerg. Infect. Dis. 26, 273-281 (2020)

15. Henritzi, D., et al.: Surveillance of European domestic pig populations identifies an emerging reservoir of potentially zoonotic swine influenza a viruses. Cell Host Microbe 28, 614-627.e6 (2020)

16. Sun, H., et al.: Prevalent Eurasian avian-like H1N1 swine influenza virus with 2009 pandemic viral genes facilitating human infection. Proc. Natl. Acad. Sci. U.S.A. 117, 17204-17210 (2020)

Open Access Dieses Kapitel wird unter der Creative Commons Namensnennung - Nicht kommerziell - Keine Bearbeitung 4.0 International Lizenz (http://creativecommons.org/ licenses/by-nc-nd/4.0/deed.de) veröffentlicht, welche die nicht-kommerzielle Nutzung, Vervielfältigung, Verbreitung und Wiedergabe in jeglichem Medium und Format erlaubt, sofern Sie den/die ursprünglichen Autor(en) und die Quelle ordnungsgemäß nennen, einen Link zur Creative Commons Lizenz beifügen und angeben, ob Änderungen vorgenommen wurden. Die Lizenz gibt Ihnen nicht das Recht, bearbeitete oder sonst wie umgestaltete Fassungen dieses Werkes zu verbreiten oder öffentlich wiederzugeben.

Die in diesem Kapitel enthaltenen Bilder und sonstiges Drittmaterial unterliegen ebenfalls der genannten Creative Commons Lizenz, sofern sich aus der Abbildungslegende nichts anderes ergibt. Sofern das betreffende Material nicht unter der genannten Creative Commons Lizenz steht und die betreffende Handlung nicht nach gesetzlichen Vorschriften erlaubt ist, ist auch für die oben aufgeführten nicht-kommerziellen Weiterverwendungen des Materials die Einwilligung des jeweiligen Rechteinhabers einzuholen. 